\title{
CAI for Minimizing Movement of Solar Tracking Concentrators
}

\author{
Carlos Ramírez, Héctor García, Livier Serna, and Noel León \\ Center of Innovation in Design \& Technology, Tecnológico de Monterrey \\ Eugenio Garza Sada \# 2501, México \\ carlos.alejandro@itesm.mx
}

\begin{abstract}
Computer-Aided Innovation (CAI) has proven useful for creative problem solving, particularly when faced with opposing restrictions. The work presented is faced with such restrictions in terms of solar tracking concentrators. The targeted low cost regarding the tracking system is opposed to the need of a constant precise and controlled movement. The literature revised will be discussed and then areas of opportunity found. The proposal involved the use of computer simulation for concept selection and validation trough a ray-trace method and mathematical models interrelationship for minimizing movement of a solar tracking concentrator. Trace Pro software from Lambda Research is used for ray-trace simulation. This method consists on the projection of a large number of rays over the optical system, and even by reflection or refraction, system's efficiency can be determined. In the end, an optimal solution is found depending on the conditions considered and conceptual designs are proposed.
\end{abstract}

Keywords: CAI, ray-trace method, iteration, solar tracking concentrator, DE system, semi-passive movement, blind system.

\section{Introduction}

In this paper, a situation having opposing restrictions is presented in terms of a solar tracking system for solar radiation concentration. A solar concentrating system consists mainly of an absorber, a receiver and a sun tracker. The absorber, which is usually a parabolic dish, has to track to the sun position throughout the day, and even throughout the year. Moreover, the initial position of the tracking system should be calibrated to the particular position on the Earth's surface due to latitude variation. One of the main problems related with the re-positioning of the concentrating system is the weight. The absorber and receiver can sometimes weight over 1,000 kilograms, depending on the size of the dish, the supporting structure and the amount of material contained in the receiver that needs to be heated.

The function of a tracking system is to enable the solar radiation to be concentrated in a limited and well-defined receiver's area. The concentration raises the temperature and the thermal energy may be stored and or transformed to other types of energy. The solar radiation concentration system should consume as little energy as possible for solar tracking.

D. Cavallucci, R. De Guio, and G. Cascini (Eds.): CAI 2011, IFIP AICT 355, pp. 43-56, 2011.

(C) IFIP International Federation for Information Processing 2011 


\section{Nomenclature}

\begin{tabular}{|c|c|}
\hline $\mathrm{Aa}$ & absorber area $\left(\mathrm{m}^{2}\right)$ \\
\hline $\mathrm{Ar}$ & receiver area $\left(\mathrm{m}^{2}\right)$ \\
\hline $\mathrm{a}_{0}, \mathrm{a}_{1, \mathrm{k}}$ & dimensionless constants \\
\hline$\beta$ & solar zenith angle \\
\hline $\mathrm{C}$ & concentration ratio \\
\hline $\mathrm{C}_{\mathrm{e}}$ & effective concentration ratio \\
\hline $\mathrm{C}_{1}, \mathrm{C}_{2}, \mathrm{C}_{3}$ & variables that allows the calculation of $\gamma$ \\
\hline $\mathrm{F}$ & furnace factor \\
\hline $\mathrm{H}$ & monthly average solar radiation $\left(\mathrm{J} / \mathrm{m}^{2}\right)$ \\
\hline $\mathrm{H}_{0}$ & local average extraterrestrial radiation $\left(\mathrm{J} / \mathrm{m}^{2}\right)$ \\
\hline $\mathrm{I}_{\mathrm{dir}}$ & terrestrial solar irradiance $\left(\mathrm{W} / \mathrm{m}^{2}\right)$ \\
\hline $\mathrm{I}_{\mathrm{ext}}$ & extraterrestrial solar irradiance $\left(\mathrm{W} / \mathrm{m}^{2}\right)$ \\
\hline $\mathrm{I}_{\mathrm{sc}}$ & solar constant $\left(\mathrm{W} / \mathrm{m}^{2}\right)$ \\
\hline $\mathrm{n}$ & day number of the year \\
\hline $\mathrm{S}$ & monthly average hours of sunlight \\
\hline $\mathrm{S}_{0}$ & monthly average day duration (hours) \\
\hline $\mathrm{T}_{\mathrm{r}}$ & receiver temperature $(\mathrm{K})$ \\
\hline$\varphi$ & earth latitude \\
\hline$\delta$ & earth declination \\
\hline$\tau_{\text {dir }}$ & atmospheric transmittance $(\%)$ \\
\hline$\sigma$ & Stefan-Boltzman constant $\left(\mathrm{W} / \mathrm{m}^{2} \mathrm{~K}^{4}\right)$ \\
\hline$\omega$ & angular displacement of the sun to east or west with respect to the local \\
\hline & meridian \\
\hline$\gamma$ & solar azimuth angle \\
\hline$\gamma^{\prime}$ & pseudo solar azimuth angle \\
\hline
\end{tabular}


For achieving a low cost tracking system the following contradiction has to be solved: on one hand the solar tracking system has to follow the sun movement. On the other hand, it is desired that the system does not move in order to not require the use of energy and therefore to minimize cost. The reconciliation of these opposing requirements has been understood as a problem that needs an innovative perspective.

The literature shows efforts that have been mainly directed to find more refined algorithms and sensing methods for tracking the solar movement. However, only limited attention has been paid to the rest of the tracking system (i.e. the structure, the materials used, and the architecture of the object). We have found only few, yet useful, works aimed to find ways to reduce the need to move on Gottsche et al., 2005 [12]; Kribus and Ries, 2002 [15].

\subsection{CAI}

The work presented at this paper follows an approach of Computer-Aided Innovation (CAI), which has proven useful for creative problem solving particularly when faced with opposing restrictions, such as the ones established above. It aims at the optimization of solar tracking systems by minimization of active movement, but taking cost requirements as also the amount of energy collected in account. A method for solving the problem is presented that enables the exploration of innovative solutions aided by computational tools. These latter have facilitated the expansion of creative exploration, the global interdependence of the system's components, and the simulation of different scenarios. Without the aid of computers, calculations and modeling would have taken longer periods of time, limiting the amount of ideas that can be tested as well as the time dedicated to imagine creative alternatives, therefore it may be claimed that a computer-aided innovation process has been applied. The contributions include but are not limited to: problem analysis acceleration and solution exploration enhancement.

Computer aid has gradually permeated the whole product design and development cycle. From the initial Computer-aided Design (CAD) and Computer-aided Engineering (CAE), the exploitation of machine calculation power has extended to the realms of innovation. Computer-aided Innovation aims at helping in tackling the problems related with innovations failures. According to Dorr et al., CAI is not limited to the use of personal computers within the innovation process, or the use of innovation tools such as TRIZ in computerized environments. It is rather the adoption of advanced information and communication methods and tools aimed at integration, virtualization, multi-scalable and multicultural collaboration [9]. The intention of these tools is to support the creative phases of design in various meaningful ways. One of them is helping the designer to model different ideas in a virtual environment and in relatively short periods (as opposed to the manufacturing of prototypes for each new idea imagined). This results in important reductions in costs in the conceptual phases of product design. Another advantage that has lately been valued is the possibility of creating new options in ways that weren't imagined before. According to Albers et al., computational tools can help in the generation of new solutions emerging from many disconnected ideas, and suggesting new concepts [4].

The present work explores this realm and presents the results of an innovation process that has been accompanied by computer simulation and optimization. The 
document that follows is organized in third main sections. The first one explains the generalities of a solar tracking system and the problems its design presents. It also contains a review of works done lately in terms of those problems and where areas of opportunity still lie. The second section includes our proposal, both method and application. The third one establishes the results obtained, what it can be concluded from them, as well as the achieved contributions.

\section{Solar Tracking Concentrators Generalities}

A solar concentrating system consists mainly of an absorber, a receiver and a sun tracker. The absorber, which is usually a parabolic dish, has to track to the sun position throughout the day, and even throughout the year. Moreover, the initial position of the tracking system should be calibrated to the particular position on the Earth's surface due to latitude variation.

There are two main types of concentrators: two-dimensional, and threedimensional. The former concentrates the solar energy throughout a line, while the latter does it in a spot. The present work considers for the case study the architecture of the type of solar concentration system called Dish/Motor (DE) shown in Fig. 1.

In terms of the overall architecture of the system, patents found in [7], [8], [11], [13], [17], [18] and [19] can attest for the attempts of reducing or eliminating the need to adjust the system to the Sun's position. Nevertheless, a tracking system that can chase the sun rays to obtain heat is ultimately indispensable. Studies for that particular part of the system can be found in [1], [2], [3], [4], [5] and [6]. The literature has as common denominator: the optimization of the system through a reduction of the periodic movement or else through an improvement of the electro-mechanical systems that enable an appropriate orientation. Up until now, however, no concentration system has eliminated the adjustments needed to compensate the variability in the solar trajectory. As much as it can be reduced, it nevertheless impacts in a direct way in the temperatures achieved and thus the overall efficiency of the system. This works aims at the exploration of innovative ways to tackle this issue.

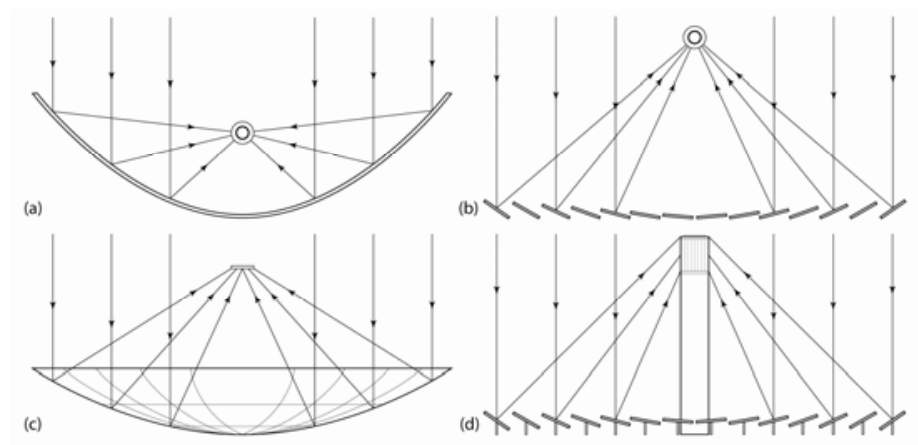

Fig. 1. Solar concentration systems. Linear concentrator (a) PTC / (b) LF, and spot concentrator (c) DE / (d) CRS 


\section{Proposal}

The work presented at this paper aims at the optimization of solar tracking systems by minimization of active movement, but taking cost requirements as also the amount of temperature collected in account. A method for solving the problem is presented that enables the exploration of innovative solutions aided by computational tools.

The methodology used in this paper consists in the interrelationship of a ray-trace method and mathematical models. This allows the evaluation of different scenarios in a short period of time without costly prototype iterations. TracePro is used for raytrace simulation, a method that consists on a projection of a large number of rays over the solar concentrator system. Mathematical models are interrelated trough spreadsheet software. These permit the performance calculation of the simulated concepts, in particular, the temperature on the receiver as this is the essential part of the concentrator's efficiency. If results are not satisfactory, iteration takes place and returns to the simulation stage. Once a scenario has been selected as a function of its movement reduction and temperature, concept is improved until results can't get better with the established system's configuration. During validation stage, physical testing is performed trough a solar concentrator scale prototype.

Mathematical models that relate solar energy variables are firstly explained. Then conceptual designs and their evaluation are presented to finally conclude with the optimization of the selected system for further validation.

\subsection{Mathematical Models}

The mathematical models allow the calculation of the main parameters of the solar concentrator system performance. These permits to visualize the behavior of different solution alternatives under ideal conditions in a short period of time such as solar motion variables, incident radiation and concentration temperature.

Since the main focus of this research is the use of CAI tools for problem solving, mathematical details are omitted and only a brief general idea of the formulas are described on the nomenclature table at the beginning of the paper. For further description of all the mathematical variables it is suggested a literature review presented in [6], [10], [14] and [16].

The apparent solar motion is one of the main variables to consider when designing a solar concentrator. Duffie and William [10] propose the following expressions to describe the sun's path

$$
\begin{aligned}
& \cos \beta=\cos \varphi \cos \delta \cos \omega+\sin \varphi \sin \delta . \\
& \delta=23.45 \sin [360(284+\mathrm{n} / 365)] . \\
& \gamma=\mathrm{C}_{1} \mathrm{C}_{2} \gamma^{\prime}+\mathrm{C}_{3}\left(1-\mathrm{C}_{1} \mathrm{C}_{2} / 2\right) 180 .
\end{aligned}
$$

The performance of any solar system not only depends on the solar tracking, but also in the amount of radiation available on Earth, thus the equations to calculate this variable are set according to Bakirci, 1961 [6]; Hottel, 1976 [14]; Liu y Jordan, 1960 [16]. 


$$
\begin{gathered}
\mathrm{H} / \mathrm{H}_{0}=0.23+0.48\left(\mathrm{~S} / \mathrm{S}_{0}\right) \\
\mathrm{I}_{\mathrm{ext}}=\mathrm{I}_{\mathrm{sc}}[1+0.033 \cos (360 \mathrm{n} / 365)] \cos \beta \\
\tau_{\mathrm{dir}}=\mathrm{a}_{0}+\mathrm{a}_{1} \exp (-\mathrm{k} / \cos \beta) \\
\mathrm{I}_{\mathrm{dir}}=\mathrm{I}_{\mathrm{ext}} \tau_{\mathrm{dir}} .
\end{gathered}
$$

Yet another variable involved is the relationship between the absorber's area and the receiver's area. The higher the ratio the higher the system's temperature. However, an $F$ variable decreases that concentration ratio due to heat losses and converts it to $C_{e}$. With this information it can be determine the temperature achieved by the concentrator. These variables are expressed through the following equations

$$
\begin{gathered}
C=A_{a} / A_{r} . \\
C_{e}=F C . \\
T_{r}={ }^{4} \sqrt{ }\left(C_{e} I_{d i r} / \sigma\right) .
\end{gathered}
$$

Notably, the case of study of the present research focuses on the installation of the system on a latitude $\varphi=25^{\circ} 39^{\prime} 15^{\prime}$ 'N, which corresponds to the city of Monterrey, N.L., México. The solar path and amount of radiation at this particular location allows the calculation of the temperature achieved by each concept in order to select the one that will need further validation.

\subsection{Computer Simulation by Ray-Trace Method}

In this section all the solar concentrator's concepts are shown. From each one of them we obtain the $T_{r}$ values using the mathematical models previously described.

The use of a computer tool to support the reduction of DE system movement due to solar position variability is the main objective of this stage. The functional analysis of DE technology involves the relationship between absorber and receiver, solar tracking system and support structure. If we require minimizing the solar concentrator movement, is therefore necessary to consider a separate system that compensate the sun's path and redirects its radiation perpendicular to the absorber's surface. Therefore Concept $A$ is proposed. In this concept, a series of reflective surfaces in a blind arrangement are placed above the concentrator. Each blind has a size of $0.1 \mathrm{~m} \times 0.5 \mathrm{~m}$, thus 19 flat surfaces are required to cover a $1 \mathrm{~m}^{2}$ absorber's area as shown in Fig. 2.

On one hand, having a stationary DE system requires less engine power for sun tracking. On the other hand, blind configuration generates shadows between each pair of reflective surface in low solar angles. Each blind blocks certain amount of radiation to its nearest neighbor, so the amount of power sent to the dish surface and temperature decreases. To avoid this, the tracking system must be tilted properly depending on $\varphi$. 

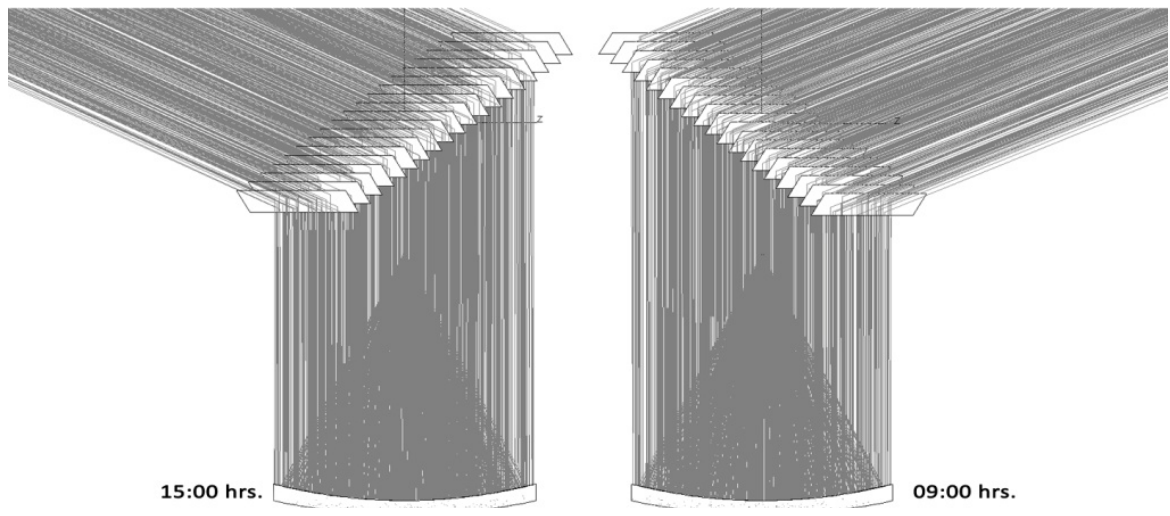

Fig. 2. Concept A

A design consideration of the tracking system is the space required for its movement. If it is desired to install the tracker on the roof of a house, it is necessary to remain the blind's framework stationary. From this perspective, Concept $B$ divides each blind into three equal segments as shown in Fig. 3. The parabolic dish and receiver remain stationary on the horizontal plane. Despite being a semi-stationary concentrator, blind segments complicate the electro-mechanical system and increase its cost. Despite this, the system has the highest amount of solar radiation collected only at noon as it covers the entire absorber's area, while in the morning and afternoon the concentrated energy decreases due to blind segments separation.
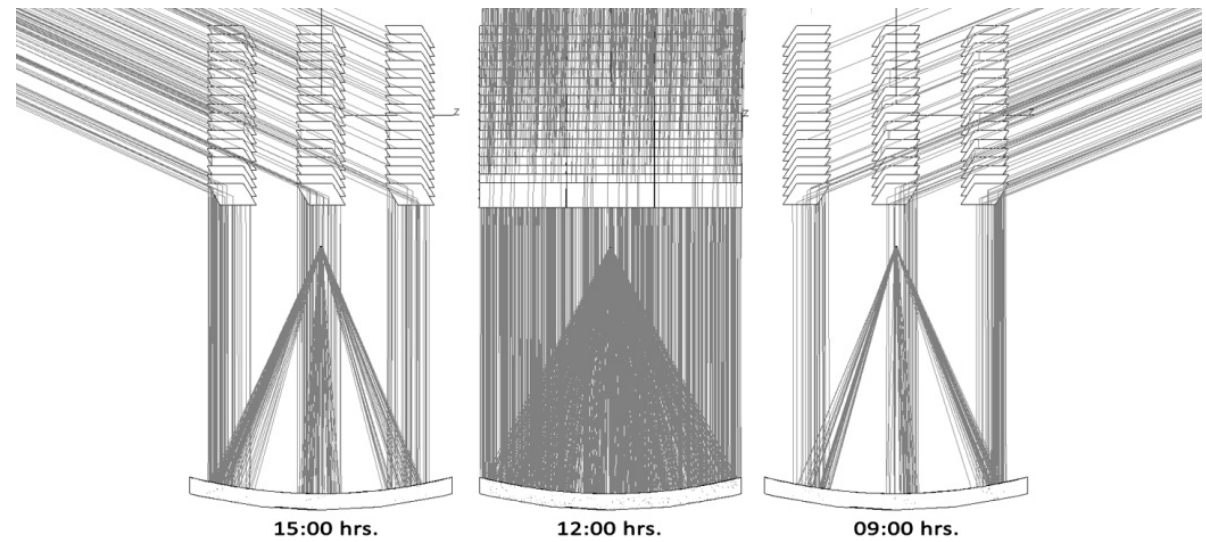

Fig. 3. Concept B

Concept $C$ showed in Fig. 4 take some of the best features of previous solutions. The system consists of a series of blinds split in half that minimize tracking while the blind's structure remain stationary and the number of components minimized. Due to absorber's orientation, the incident radiation at noon maximizes $T_{r}$ since the whole dish's area is used. The absorber and receiver must be minimally shifted throughout the year. 


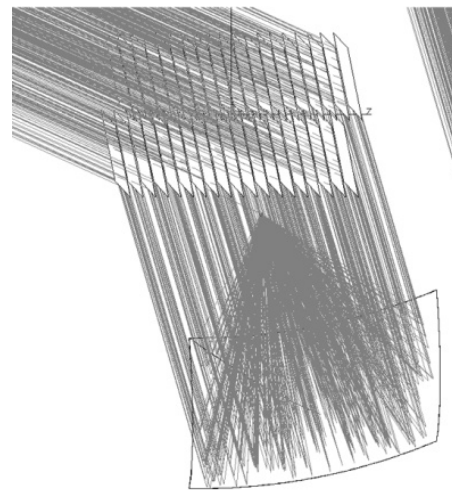

15:00 hrs.

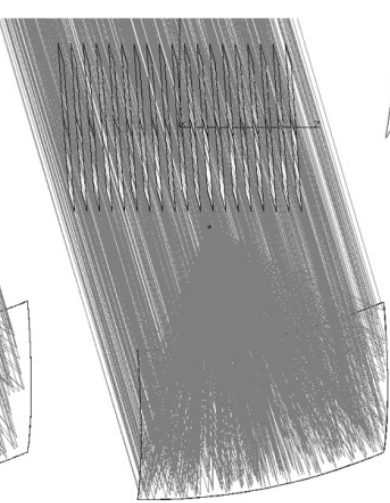

12:00 hrs.

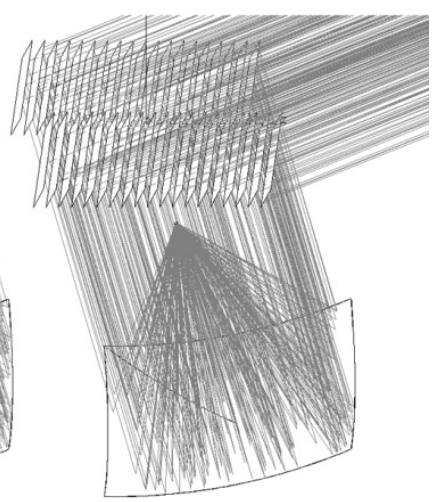

09:00 hrs.

Fig. 4. Concept $\mathrm{C}$

\subsection{Evaluation}

The evaluation of previous scenarios requires computational tools to measure the motion reduction in both azimuth and altitude angles (see Table 1) and the temperature profile (see Fig. 5). Both results allow the selection of an adequate concept for further improvement.

In order to measure the DE system movement's reduction, the solar angles $\beta$ and $\gamma$ were taken as the basis to determine the variability of the position of the each concept blinds.

Table 1. Two-axis motion made by DE system and all design concepts from 9:00 to 15:00 hrs

\begin{tabular}{ccc}
\hline Concept & Altitude & Azimuth \\
\hline DE & $24^{\circ}$ & $70^{\circ}$ \\
A & $13^{\circ}$ & $70^{\circ}$ \\
B & $32^{\circ}$ & $70^{\circ}$ \\
C & $35^{\circ}$ & $10^{\circ}$ \\
\hline
\end{tabular}

The DE system requires a two axes movement to compensate the solar angles variation and concentrate radiation on the receiver. It takes $24^{\circ}$ in altitude and $70^{\circ}$ in azimuth to achieve it, which could be difficult depending on its weight. Despite this, the system reaches the highest temperature levels comparing to other types of concentrators. By using $1 \mathrm{~m}^{2}$ with a $\mathrm{C}_{\mathrm{e}}=1000$, it is possible to achieve a temperature of $1484^{\circ} \mathrm{C}$ at noon on $\varphi=25^{\circ} 39^{\prime} 15^{\prime \prime} \mathrm{N}$.

Concept $A$ shows a 54\% decrease in altitude movement and remains constant on azimuth compared to DE system. Despite this, blinds must have enough space to achieve this motion as their configuration suggests the reorientation of its whole structure. The temperature profile is affected at noon due to shadow generation between blinds.

The motion carried by the $D E$ system increases in Concept $B$. However, these latter requires a stationary dish and receiver, thus eliminating majors efforts for sun 
tracking. Finally, Concept $C$ shows a $46 \%$ increase of movement in altitude and a decrease of $86 \%$ in azimuth compared to DE system. Beside this, the temperature profile has a value of $1484^{\circ} \mathrm{C}$ at noon on May and July, which equals the maximum temperature of the DE system. It is therefore appropriate to think that Concept $C$ is ideal for solving the research problem; however, it requires design improvement in order to raise the temperature profile around noon.

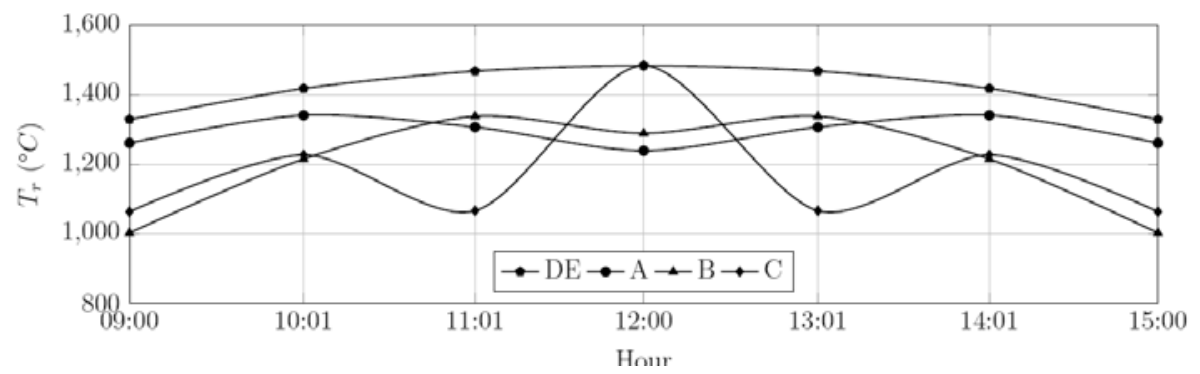

Fig. 5. $T_{r}$ reached by all design concepts on May/July (maximum $T_{r}$ )

\subsection{Concept Improvement}

This section discusses the conceptual improvement process for Concept $C$ previously selected following a CAI approach. The main objective is to collect the maximum amount of solar radiation around noon to maximize the temperature reached by the system. Low temperature profile is due to decrease of the blinds visible area with respect to the sun around noon, which reduces the effective area of the absorber and thus the concentration ratio.

A possible solution is to increase the visible blinds surface at noon; which means increasing its quantity inside a $1 \mathrm{~m}^{2}$ area. Finding the optimal dimensions and blinds spacing requires a certain iteration to achieve improved results; ray-tracer software and mathematical models are useful tools to determine quantitatively the optimum problem solution. Different blind dimensions and spacing were simulated. $T_{r}$ values achieved by these options are shown in Fig. 6.

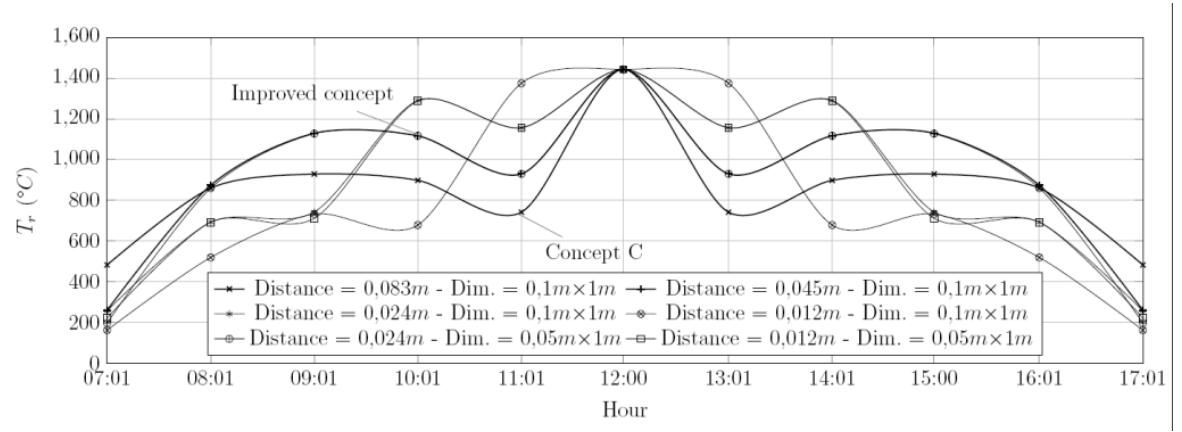

Fig. 6. $T_{r}$ reached on May/July with different distances and dimensions of solar blinds within an area of $1 \mathrm{~m}^{2}$ 
Concepts with the greatest temperature stability are the ones with blind separation of $0.045 \mathrm{~m}$ and $0.05 \mathrm{~m}$ with dimensions of $0.1 \mathrm{~m} \times 1.0 \mathrm{~m}$ and $0.05 \mathrm{~m} \times 1.0 \mathrm{~m}$ respectively. The former requires 42 blinds, while the latter 82 . Both concepts have an average temperature increment of $250^{\circ} \mathrm{C}$ from 9:00 to 15:00 hrs. with respect to the original one.
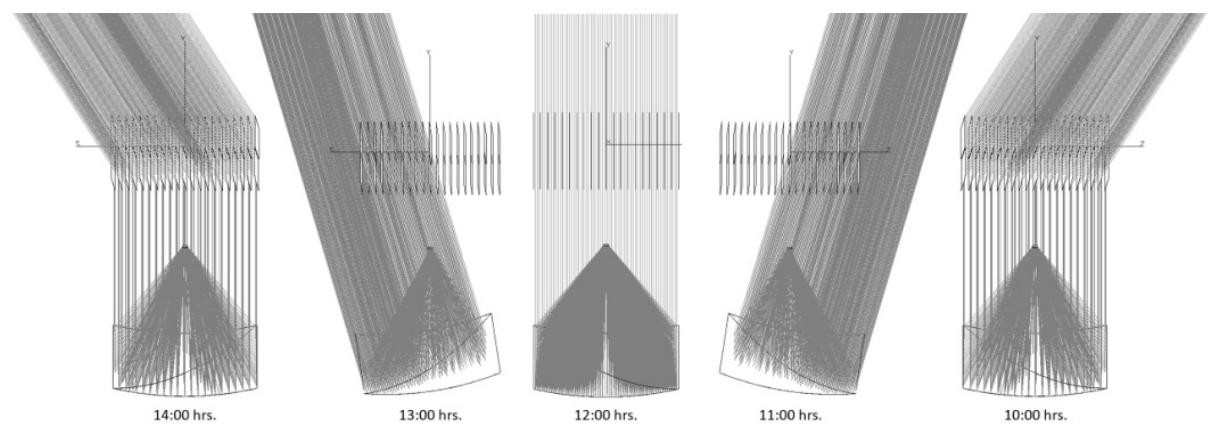

Fig. 7. Solar concentrator system with blind system and minimum movement dish

Although $T_{r}$ shows an increment with the system optimization achieved so far, there are temperature valleys at 11:00 and 13:00 hrs. On one hand, DE systems generate a constant temperature profile because they remained the perpendicularity between the dish's surface and the solar radiation throughout the day; however, periodical movement is required to do so. On the other hand, minimum tracking suggest the usage of blinds to eliminate this adjustment. Therefore, using this latter at mornings and evenings combined with a semi-passive dish at noon, the objective of $T_{r}$ maximizing can be accomplished (see Fig. 7). A temperature comparison between fixed and semi-passive absorber is shown on Fig. 8.

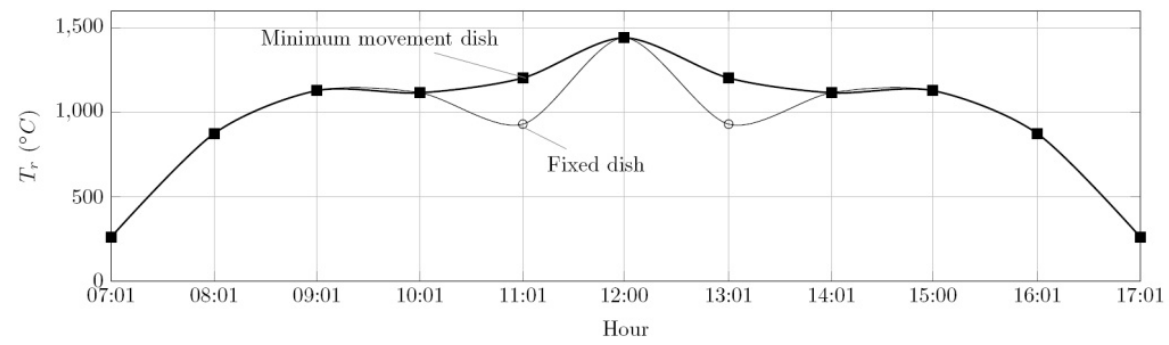

Fig. 8. $T_{r}$ reached by a semi-passive solar concentrator on March/September

\subsection{Validation}

The objective of this section is to validate the performance of the solar concentration system trough physical experimentation. For that reason, a $1 \mathrm{~m}^{2}$ scale prototype was build and proved. 
The validation stage allows verifying the simulated performance of the improved system through a prototype that resembles its operation.

The computer simulation allowed the visualization of the concept's behavior under "virtual conditions", however, some other aspects as assembly, weight, movement, and other problems arise when taking the system to real life. Besides this, it is possible to obtain tangible results by taking into account some variables unconsidered before as a calculated solar angles precision, real incident radiation and final concentration temperature due to heat losses.

A $1 \mathrm{~m}^{2}$ prototype was build because its results can be replicated when scaling as long as concentration ratio remains constant. Because it is pretended to build a $30 \mathrm{~m}^{2}$ system for home electricity generation, the concentrator could be either scaled or repeated.

The prototype comprises an architecture made of a parabolic dish and a blind system both coated with a $95 \%$ hemispherical reflection film. Blinds were assembled into three degrees of freedom structure for sun tracking throughout the day and year.. The concentration factor resulted in a ratio of 1:670 due to dish geometry. Prototype's architecture can be seen on Fig. 9.

The parameter that allowed system validation was the temperature $T_{r}$ obtained by using a high efficiency thermometer. Validation stage took place on $\varphi=25^{\circ} 39^{\prime} 15^{\prime \prime} \mathrm{N}$ in order to obtained similar results between computational simulation and real conditions operation. This latitude corresponds to Monterrey, N.L., México, which has a solar radiation average of $0.934 \mathrm{~kW} / \mathrm{m}^{2}$ during daytime as shown in Fig. 10,

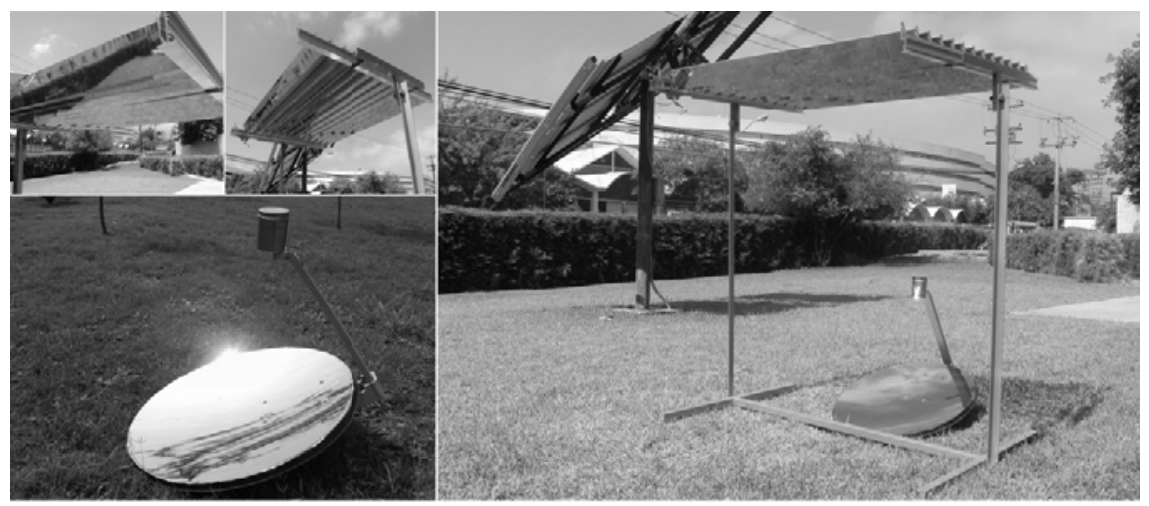

Fig. 9. Semi-passive solar concentrator prototype

$T_{r}$ results are shown in Fig. 11. It is observed that the system's temperature profile is similar to the one obtained in the concept improvement stage. In both graphs, $T_{r}$ valleys are eliminated by moving the dish around noon hours. 


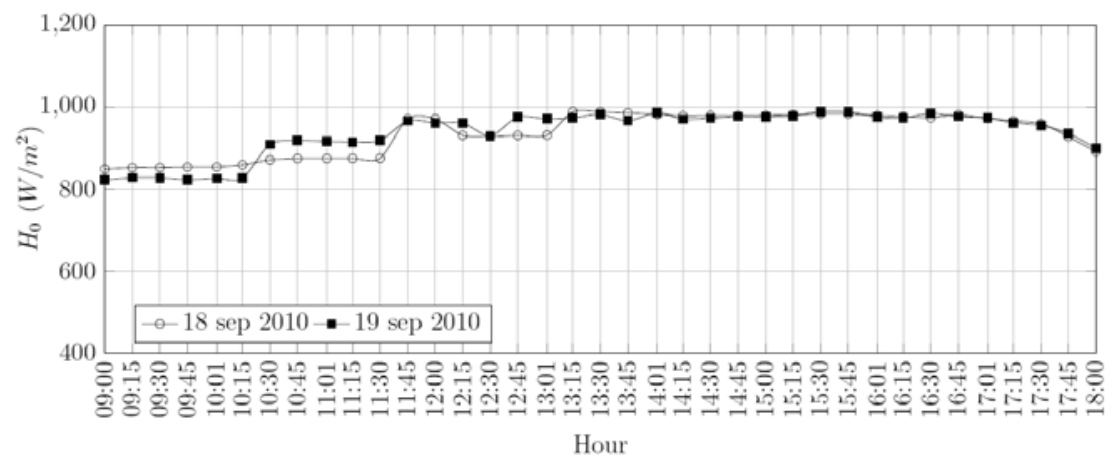

Fig. 10. $\mathrm{H}_{0}$ on $\varphi=25^{\circ} 39^{\prime} 15^{\prime \prime} \mathrm{N}$

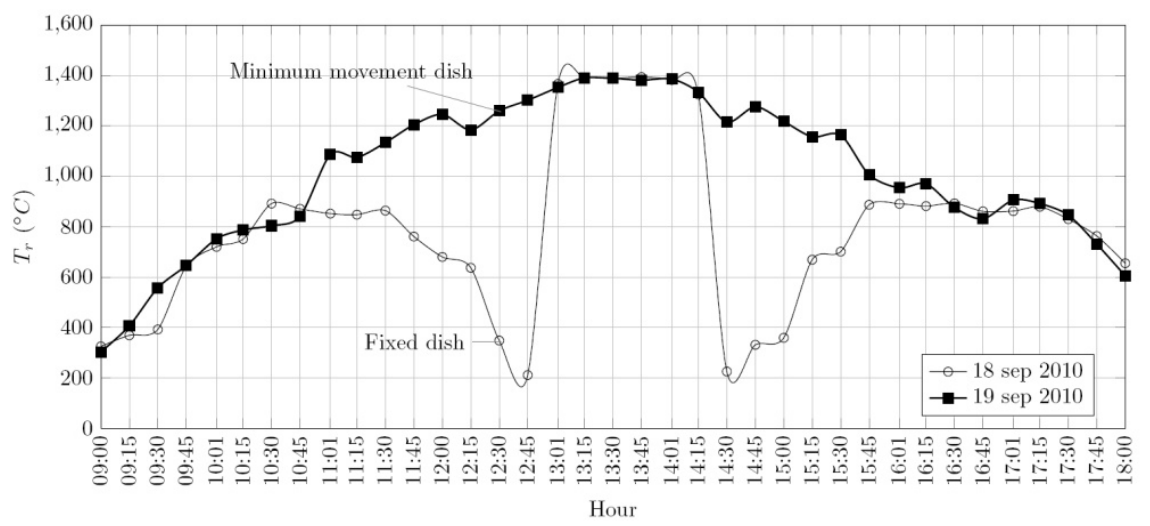

Fig. 11. $T_{r}$ reached by semi-passive solar concentrator prototype

\section{Results and Contributions}

This research solved the resultant contradictions through a CAI approach by means of computational tools that helped to reduce DE concentrator movement required to follow sun's path. Optimal results were obtained by iteration method that related raytrace tool with mathematical models. On one hand the ray-tracer helped to visualize the different concepts that would lead to the solution. On the other, the results that allowed the concept selection were possible due to calculus software that permitted a quick and precise interrelation between several mathematical variables, making the development a greater number of alternatives possible.

A relationship between CAI and an iteration methodology was proposed. Through a series of steps, this helped to find an optimal solution in a short period of time. The concept simulation was the first step of the research, were different solar concentrators systems were modeled in CAD software and simulated in Trace Pro. Without this tool, the rapid and accurate conceptual exploration performed wouldn't have been possible. The complement of the simulation was the solution of 
mathematical models with calculus software that allowed the evaluation of temperatures as a function of solar movement and amount of incident radiation. As a case of study, values corresponding to $\varphi=25^{\circ} 39^{\prime} 15^{\prime \prime} \mathrm{N}$ were used. While the expected results were not achieved in the previous stage, concept improvement allowed adjusting certain parameters under CAI approach. Finally, validation stage allowed the construction of a prototype in order to verify its functionality under real conditions. Although the use of computational tools was omitted at this point, the prototype wouldn't have been possible without a previous virtual simulation and evaluation.

Accordingly, it can be established that CAI approach promotes problem solution in a fast and creative way. Its relationship with an iterative methodology led to the development of a semi-passive solar concentrator in a short period of time and at low cost.

\section{Conclusions}

A method for solving the problem is presented that enables the exploration of innovative solutions aided by computational tools. These latter have facilitated the expansion of creative exploration, the global interdependence of the system's components, and the simulation of different scenarios. Without the aid of computers, calculations and modeling would have taken longer periods of time, limiting the amount of ideas that can be tested as well the time dedicated to imagine creative alternatives.

\section{Acknowledgments}

To Ph.D. Livier Serna and Ph.D. Noel León for their invaluable research assistance.

\section{References}

1. Abouzeid, M.: Use of a reluctance stepper motor for solar tracking based on a programmable logic array (PLC) controller. J. of Renewable Energy 23, 551-560 (2001)

2. Abu-Khader, M., Badran, O., Abdallah, S.: Evaluating multi-axes sun-tracking system at different modes of operation in Jordan. J. of Renewable and Sustainable Energy Reviews 12, 864-873 (2008)

3. Al-Soud, M., Abdallah, E., Akayleh, A., Abdallah, S., Hrayshat, E.: A parabolic solar cooker with automatic two axes sun tracking system. Applied Energy 87, 463-470 (2010)

4. Albers, A., Leon, N., Aguayo, H., Maier, T.: Optimization with Generic Algorithms and Splines as a way for Computer Aided Innovation. In: IFIP International Federation for Information Processing, vol. 277, pp. 7-18 (2008)

5. Arbab, H., Jazi, B., Rezagholizadeh, M.: A computer tracking system of solar dish with two-axis degrees of freedoms based on picture processing of bar shadow. J. of Renewable Energy, 1114-1118 (2009) 
6. Bakirci, K.: The estimation of monthly mean values of daily total short wave radiation on vertical and inclined surface from sunshine records for latitudes 40N-40S. In: Proceedings of UN Conference in New Sources of Energy, pp. 378-390 (1961)

7. Boyd, J.: Radiation Concentrator. US Patent 4552126 (1984)

8. Bunch, J.: Heliostat apparatus. US Patent 4110009 (1978)

9. Dorr, N., Behnken, L., Muller-Prothmann, T.: Web-based Platform for Computer Aided Innovation. In: IFIP International Federation for Information Processing, vol. 277, pp. 229-237 (2008)

10. Duffie, J., William, A.: Solar engineering of thermal processes. Wiley, New York (1991)

11. Fletcher, C., Perkins, G.: Sun tracking solar energy collector. US Patent 4111184 (1978)

12. Gottsche, J., Hoffschmidt, B., Schmitz, S., Sauerborn, M.: Solar concentrating systems using small mirror arrays. J. of Solar Energy Engineering 132 (2010)

13. Gross, W., Luconi, G., Zsolnay, D.: Solar concentrator array with individually adjustable elements. US Patent 6959993 B2 (2005)

14. Hottel, H.: A simple model for estimating the transmittance of direct solar radiation through clear atmospheres. J. of Solar Energy 129 (1976)

15. Kribus, A., Ries, H.: LiMoNAED: A limited motion, non-shading, asymmetric, ecliptictracking dish. J. of Solar Energy 73, 337-344 (2002)

16. Liu, B., Jordan, R.: The interrelationship and characteristic distribution of direct, diffuse and total solar radiation. J. of Solar Energy 1 (1960)

17. O'Neill, M.: Color-mixing lens for solar concentrator system and methods of manufacture and operation thereof. US Patent 6031179 (2000)

18. Sampayo, E.: Solar concentrator system. US Patent 5542409 (1996)

19. Szulmayer, W.: Solar concentrator. US Patent 4230094 (1980) 